\title{
Fast and Slow Bars: A Comparative Analysis
}

\author{
Valerij L. Polyachenko \\ Institute of Astronomy, 48 Pyatnitskaya St., Moscow 109017, Russia
}

\begin{abstract}
The in-plane appearance of barred galaxies is formed in either of two ways: 1) the usual bar-mode instability in a fast rotating disk forms a fast bar, while 2) the radial orbit instability (or its modifications) leads to a slow bar. The vertical structure depends on the universal mechanism of the bending instability both in fast and slow bars. Among all the relevant topics, I chose (i) a critical review of the work on the bending instability, and (ii) some comments concerning the local and global stability criteria for non-axisymmetric disturbances in galactic disks; in particular, a new approximate local dispersion relation for a star disk is presented. Also shown is a range of accuracy for the intuitional global criterion of the slow bar instability of the Ostriker-Peebles type (on the example of the exactly-solved model).
\end{abstract}

\section{Introduction}

Generally speaking, the formation of bars, with all their complex 3D-arrangement, need not be a result of instabilities, much less of those highly simplified versions commonly used. Nonetheless, the study of these instabilities and the structures which they produce is indubitably useful (for more detail, see Fridman \& Polyachenko 1984 (henceforth FP); Sellwood \& Wilkinson 1993; Polyachenko 1994).

There are few basic instabilities which could play an important part in the bar formation processes (the full list of instabilities in stellar systems is poor enough too, e.g., compared with plasma). Namely: first, the bar-forming (in a narrow sense) instability responsible for the bar's appearance in the plane of rotation (top view), and, second, the bending instability which determines the bar's side view. Following are brief discussions of these instabilities and their connections with galactic bars.

As is clear from the names, fast and slow bars differ first in their pattern speeds; accordingly, a fast bar ends near the corotation resonance (CR) while a slow (or moderate ${ }^{1}$ ) bar is of order of the inner Lindblad resonance (ILR) radius in size.

While there are several different ways for in-plane bar formation, the only physical mechanism for thickening the flat stellar systems is, as yet known, just that of the instability which is called 'bending'; the simplest mechanical

\footnotetext{
${ }^{1}$ The convenient classification of bars was described by Pasha and Polyachenko (1994).
} 
analogy of this instability is the behavior of a metallic ruler compressed on its edges. Conceptually ${ }^{2}$, the bending instability acts identically in both fast and slow bars, and I begin a more detailed presentation from this instability (Sec. 2), somewhat departing from a common practice. Such a change of the usual order is also justified because just the bending instability has recently become the subject of extensive study (e.g., Merritt \& Sellwood 1994). The study was provoked by some new interesting applications of the bending instability: e.g., this instability is possibly to be important in the formation of the specific, 'peanut' bar shape (e.g., see Raha et al. 1991; the bending instability by itself in bars is not, of course, surprising). It is convenient to follow the chronology in presenting the principal results; in passing, I correct some inexactitudes made in the bending instability presentation by Merritt \& Sellwood (1994). Section 3 considers the bar-forming instabilities (Subsection 3.1 is devoted to fast bars, Subsection 3.2 to slow bars).

\section{Bending instability}

Bending (or hose, hose-pipe, fire-hose) instability was discovered by Toomre (1966) and re-discovered by Kulsrud, Mark \& Caruso (1971). These two papers were carried out independently (despite five years of delay with the second article) because Toomre's article was published in an obscure summer-school proceedings. Such a 'semi-publication' of that paper presumably resulted from Toomre's skepticism: he did not think that this instability can be of much interest (so the majority of workers have not seen that paper up to the present, and we all know it only in its recent presentation by Merritt \& Sellwood (1994)). In this case, however, Toomre's skepticism is easy to understand: in the sixties, any flattening of stellar systems (including elliptical galaxies!) was attributed to rotation; as for disk galaxies which have always been the subject of his highest interest, they were thought to be 'cold' everywhere.

The two papers (by Toomre and by Kulsrud et al.) are almost identical in their results:

1. They derived the dispersion relation for a sheet, i.e. a layer of negligible thickness in $z$ and uniform in $x$ and $y$ :

$$
\omega^{2}=2 \pi G \sigma k-k^{2} v_{y}^{2}
$$

where $v_{y}$ is the velocity dispersion of stars in any one direction parallel to the plane, $k$ is the disturbance wavenumber, and $\sigma$ is the surface density. This equation shows that there occurs the instability $\left(\omega^{2}<0\right)$, for sufficiently short wavelengths.

2. They used the same considerations for estimating a critical value of anisotropy, i.e. of the ratio $v_{z} / v_{y}\left(v_{z}\right.$ is the velocity dispersion normal to the plane), when the bending instability of a slab (a layer of a finite thickness $2 h$ )

\footnotetext{
${ }^{2}$ It is evident from the physics of the bending instability that 'hot' slow bars should be thicker than 'cold' fast bars, all things being equal (first for the same halo mass). But bending instability phenomena for both types of bars have much in common: stabilization by a halo or a central mass, peanut or box shapes, etc.
} 


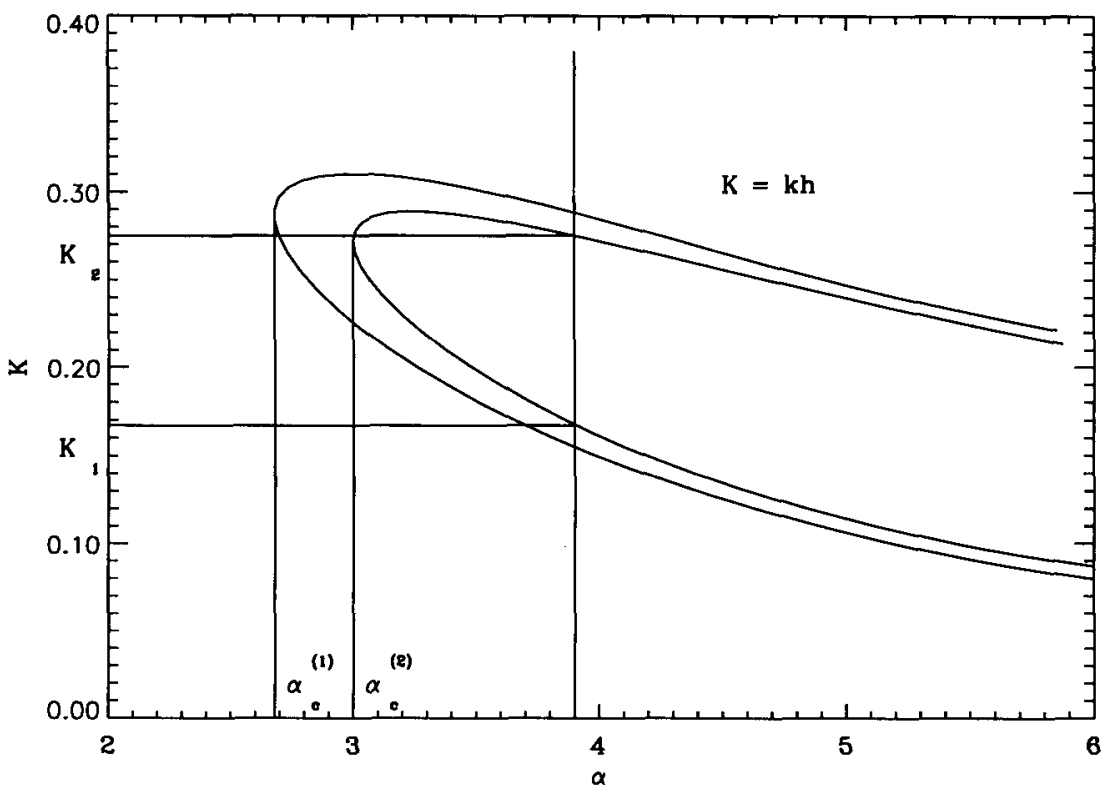

Figure 1. The curves of the bending instability boundary for star slabs

was suppressed. Namely: appealing to intuition, they claimed without ceremony that the slab should be stable for wavelengths comparable to its thickness. Of course, such an estimation could not claim to be exact. However, Kulsrud et al. mentioned that "Toomre has found in a numerical investigation that the instability only occurs for $v_{z}^{2} / v_{y}^{2}<0.1$ ", i.e. for $v_{z} / v_{y}<0.316$. They continue: "so it would appear difficult for such an instability to occur." Thus, I may note also that

3. They all were unanimous in their sceptical relation to this instability. The bending instability of the slab was studied by Polyachenko \& Shukhman (1977); for simplicity, we considered the model of the slab uniform in density; the outer curve in Figure 1 is the instability boundary we found (a very similar curve was recently published by Merritt \& Sellwood 1994). Our analytical theory of 1977 was exact for a sufficiently thin slab, when $v_{z} / v_{y}=1 / \alpha<<1$; for larger values of this ratio, the theory was approximate. In Figure 1, the exact instability boundary ${ }^{3}$ shows the inner line; this figure is taken from FP. According to the dispersion relation (1), the instability occurs for perturbations with wavelengths $\lambda<\lambda_{1}, \lambda_{1} \approx h \alpha^{2}$. In fact, one can see from Figure 1 that, for each given slab (fixed dispersion ratio ), there are two limiting wavelengths: 1) Toomre's and 2) still one boundary, from the short-wavelength side, $\lambda>\lambda_{2}, \lambda_{2} \approx h \alpha$, disturbances with shorter wavelengths are damping. The instability region narrows

\footnotetext{
${ }^{3}$ This curve was a result of standard compntations with the use of the expansion of the perturbed potential over the complete system of functions (Legendre polynomials).
} 
with decreasing $\alpha$ and vanishes at some critical $\alpha=\alpha_{c}$. It occurs at $\alpha_{c}=2.68$, in the approximate theory; the computed exact $\alpha_{c}=3.0$. Correspondingly, the inverse values which Toomre used are:

$$
\begin{aligned}
& \left(v_{z} / v_{y}\right)_{c} \approx 0.373, \text { in approximate calculations, } \\
& \left(v_{z} / v_{y}\right)_{c} \approx 0.333, \text { in exact computations. }
\end{aligned}
$$

Recall that Toomre's N-body estimation was $\left(v_{z} / v_{y}\right)_{c} \approx 0.316$. Araki (1985) showed that the above result (2) depends slightly on the model used: for the anisotropic Maxwellian velocity distribution, i.e. in the $\operatorname{sech}^{2}(z)$-model for the vertical density profile, he found ${ }^{4}:\left(v_{z} / v_{y}\right)_{c} \approx 0.293$. Rather detailed analysis of the physical mechanisms of this instability and its stabilization is available in our papers (Polyachenko \& Shukhman 1977 1979) and the monograph (FP). When moving up in the vertical straight-line in Figure 1, decreasing progressively the disturbance wavelength, we enter the instability region at $k=k_{1}$, here $\left(k>k_{1}\right)$ the instability being of the hydrodynamic nature: all particles take part in the instability process simultaneously and coherently; we leave the instability region at $k=k_{2}$, where

$$
\lambda / v_{y} \approx 1 / \omega_{z}
$$

(or $k v_{y} \approx \omega_{z}, \omega_{z}$ is the frequency of free oscillations of particles in the equilibrium slab), here $\left(k<k_{2}\right)$ the instability being of the kinetic nature: only a narrow group of resonance particles $\left(k v_{y} \approx \omega_{z}\right)$ takes part in the growth, at $k<k_{2}$, or in the decay, at $k>k_{2}$, of an initial perturbation. (The same condition (in essence) occurs, for example, for Jeans instability.) In the limit of very short waves, $\lambda / v_{y}<<1 / \omega_{z}$, a particle during the time of one free vertical oscillation crosses many waves; the corresponding perturbations are damping. By the way, the term 'Landau damping' in its original sense (Landau 1946) concerns just such short-wavelength disturbances. When turning to the global modes in finite systems, we should first replace $\lambda$ in formula (3) by the system's radius $R$; then $\lambda / v_{y} \approx c_{1} R / v_{y} \approx c_{2} / \omega_{y} \approx 1 / \omega_{z}$, where $\omega_{y}$ is the characteristic frequency of star oscillations, and $c_{1}$ and $c_{2}$ are constants. So, the condition for the instability boundary should be expressed in terms of the ratio of star frequencies in perpendicular directions. Our following stability study of homogeneous oblate and prolate spheroids (Polyachenko \& Shukhman 1979, see also FP) confirmed such a hypothesis. The behavior of the instability boundary curve $\gamma^{2}=\gamma^{2}(c / a)(\gamma \Omega$ is the spheroid's (as a whole) angular velocity of rotation, $\Omega$ is the star frequency in the $(x, y)$-plane, $c / a$ is the axis ratio) is especially simple for the bell-shaped mode of the oblate spheroid (see, e.g., Figure 53a in FP): it is the straight line beginning at $\gamma^{2} \approx 0.25$ (for $c / a=0$ ) up to $\gamma^{2} \approx 0.6$ at $c / a=0.3$, where this curve suddenly dropped; the system is stable for $c / a>0.3$. The behaviors of the marginal curves for other modes are similar, and the same is true for prolate spheroids (see FP, p. 313). The sharp decrease of the curves occurs at such a thickness, for which in the homogeneous model used there are resonances between the star oscillation frequencies with respect to $z$ and the frequencies in

\footnotetext{
${ }^{4}$ Merritt \& Sellwood (1994) mistakenly stated that Araki's paper was the first study of the bending instability in a slab (missing our earlier work).
} 
the rotation plane $(x, y)$. For the bell mode, this is the resonance $\omega_{z}=2 \Omega$; just this mode is the most 'dangerous'; for the $m=1$ mode, the resonance is $\omega_{z}=3 \Omega$, and so on. Such a strong influence of these resonances is due, of course, to the idealization of the model. For the real nonhomogeneous systems, the curves will have a smoother form. One can note a natural consequence of this result. Let us imagine a stellar system (bar, bulge, ...) formed from an initially sufficiently thin proto-system as a result of saturation of the bending instability. Then the average ratio of star oscillation frequencies is likely to be about $2: 1^{5}$ (for instance, for the final state in Figure 3 (bottom, left) of the paper by Polyachenko \& Polyachenko (these proceedings) this ratio equals to $1.89: 1$, under the simplest way of averaging). So the importance of the resonance orbit family in a thick bar (that is emphasized by Combes et al. 1990) is possibly made due to the system coming to the boundary of the bending instability. As for an exact general criterion of the bending instability, it is likely to be impossible. It is hardly probable that any more certain criterion (than above $2: 1$ average - in what sense? - frequency ratio) exists. I could remind the preceding experience of searching the 'universal' stability criterion for the fast bar mode (beginning with the well-known paper by Ostriker \& Peebles 1973) or the radial orbit instability of spherical systems (myself); both these attempts had only a limited success. Below, I show that the same is true for the slow bar mode.

\section{Bar-forming instabilities}

\subsection{Fast bars}

Since almost all papers at this meeting are devoted to fast bars ${ }^{6}$, here I decided to restrict myself to only one of the relevant problems, namely, the problem of the instability criteria. The derivation of the general criterion for the bar-mode instability of arbitrary disks is difficult if not impossible to realize, so we are forced to use the local dispersion relations and corresponding local instability criteria $^{7}$.

Unlike the gas disks (see Goldreich \& Lynden-Bell 1965; Polyachenko \& Strel'nikov 1989; Polyachenko 1990), even the rigorous local dispersion relations for the star disks cannot exist in principle. I know two different ways for deriving the approximate local dispersion relations, both giving similar results. One of them was suggested in the diploma work of my student (Strel'nikov 1989). Let us start from the integral equation for the Fourier transformation $\Phi_{q}$ of the perturbed potential $\Phi(r)$ (that can be obtained from the corresponding equation by Julian \& Toomre 1966):

$$
\frac{c}{2} \Phi_{q} \sqrt{q^{2}+k^{2}}=-\int_{q}^{\infty} \Phi_{q^{\prime}} K\left(q, q^{\prime}\right) e^{\nu\left(q^{\prime}-q\right)} d q^{\prime},
$$

\footnotetext{
${ }^{5}$ Merritt \& Sellwood (1994) think that this is a general principle.

${ }^{6}$ True, in several papers (e.g., Combes, Elmegreen, Zasov, Noguchi), the slow/moderate bars do occur, but under the other names: ILR-bars, Lynden-Bell's bars, induced (tidal) bars, etc.

${ }^{7}$ The best known example is the Goldreich - Lynden-Bell - Toomre swing amplification local mechanism applied to the grand fast bar instability.
} 
where we assume the perturbation $(\sim \exp (i m \varphi-i \omega t))$ to be localized near the radius $r_{0}$, the star distribution function in the velocities $v_{r}, v_{\varphi}$ is $f_{0}=$ $A \exp \left(-Q_{0}\right), A=\sigma_{0} \Omega_{0} / \pi \kappa c_{r}^{2}, Q_{0}=\left(v_{r}^{2} / c_{r}^{2}+v_{\varphi}^{2} / c_{\varphi}^{2}\right) / 2, \sigma_{0}=\sigma(r=0), \sigma(r)$ is the surface density, $\Omega_{0}=\Omega(r=0), \Omega(r)$ is the disk angular speed, $\kappa$ is the epicyclic frequency, $c=\kappa^{2} \Delta / \pi G \sigma_{0}, k=k_{\varphi} \Delta, k_{\varphi}=m / r_{0}, \Delta=\kappa / m\left|\Omega^{\prime}\right|, \Omega^{\prime}=$ $d \Omega / d r, \nu=\gamma / \kappa, \gamma=\operatorname{Im}(\omega), K\left(q, q^{\prime}\right)=h\left(q, q^{\prime}\right) \exp \left(-x g\left(q, q^{\prime}\right)\right), x=c_{r}^{2} / 2 \kappa^{2} \Delta^{2}$, $h\left(q, q^{\prime}\right)=\left(q q^{\prime}+\alpha^{4}\right) \sin \left(q-q^{\prime}\right)-\alpha^{2}\left(q-q^{\prime}\right) \cos \left(q-q^{\prime}\right), g\left(q, q^{\prime}\right)=q^{2}+q^{\prime 2}+2 \alpha^{4}-$ $2\left[\left(q q^{\prime}+\alpha^{4}\right) \cos \left(q-q^{\prime}\right)+\alpha^{2}\left(q-q^{\prime}\right) \sin \left(q-q^{\prime}\right)\right.$, where $\alpha^{2}=2 \Omega_{0} / r_{0}\left|\Omega_{0}^{\prime}\right|, k^{2}=$ $\alpha^{4}-\alpha^{2}$. Multiplying equation (4) by $e^{i q x}$, integrating with respect to $q$ between $q=-\infty$ and $q=+\infty$, reversing the order of the integrals in $q$ and $q^{\prime}$, we find

$$
\int_{-\infty}^{\infty}\left[\frac{c}{2} \sqrt{q^{2}+k^{2}}+\int_{-\infty}^{\infty} e^{i\left(q-q^{\prime}\right) z} K\left(q, q^{\prime}\right) d q^{\prime}\right] \Phi_{q} e^{i q x} d q=0
$$

where $z=\left(\omega-m \Omega_{0}\right) / \kappa$. For deriving the dispersion relation, let us consider a single harmonic: $\Phi_{q}=\delta\left(q-q_{0}\right)$; then we obtain a desired result:

$$
\frac{c}{2} \sqrt{q^{2}+k^{2}}+\int_{0}^{\infty} e^{i t z} H(t, q) d t=0,
$$

where $H(t, q)=h(t, q) e^{-x g(t, q)}, h(t, q)=\alpha^{2} t \cos (t)-\left(q^{2}-q t+\alpha^{4}\right) \sin (t), g(t, q)=$ $-2 \alpha^{2} \operatorname{tsin}(t)+2\left(q^{2}-q t+\alpha^{4}\right)(1-\cos (t))+t^{2}$.

The criterion of the marginal stability is obtained from (6) at $z=0$ : then we have the dependence $Q_{0}=Q_{0}(\lambda)$ where $Q_{0}=\kappa c_{r} / 2 \pi G \sigma_{0}, \lambda=c / 2 \sqrt{q^{2}+k^{2}}$. In principle, the behavior of these curves is similar to that for the gas case (e.g., Polyachenko 1990); in particular, here we also have some value of $Q_{0}=$ $Q_{m}\left(Q_{m}^{2} \approx 3\right)$ which guarantees that perturbations may not grow when $Q_{0}>Q_{m}$. This result agrees with that obtained by the second method of deriving the approximate local dispersion relation (Toomre 1981). A detailed presentation of the results of analyzing the relations above can be found in our paper (Polyachenko \& Strel'nikov 1995).

\subsection{Slow bars}

Referring for a detailed discussion of this subject to our papers (Polyachenko 1989, 1992; Pasha \& Polyachenko 1994; Polyachenko \& Polyachenko 1994, 1995, see also our paper in these proceedings), I will enlarge here on the particular problem of formulating the general instability criteria for large-scale modes.

As is well-known, Ostriker \& Peebles (1973) were the first who attempted to suggest the hypothesis, according to which stability or instability of an isolated axial-symmetrical system (fast bar-mode) is defined by the ratio $t=T_{\text {rot }} /|W|$, where $T_{\text {rot }}$ is the kinetic energy of rotation and $W$ is the potential energy. There exists a large body of critical remarks concerning this criterion (see especially Toomre 1981); nonetheless, it is operable in many cases. The Ostriker-Peebles criterion was based only on intuition because any simple model suitable for the analytical study is as yet unknown. By the way, it is concerned with the fact that the fast bar-mode instability is easily saturated by a modest halo mass. In contrast, the slow bar-mode instability cannot be stabilized by any finite halo mass, and just in the limit of large halo-to-disk mass ratios $M_{h} / M_{d}$, the 
stability problem can be solved analytically (Polyachenko 1989, 1992). It allows for comparing the exact instability criterion with that suggested by intuition. The last-mentioned criterion is, in this case, $\bar{t} \equiv T_{p r} /\left|W_{G}\right|<\overline{t_{c r}}$, where $T_{p r}$ is the part of the kinetic energy of the active subsystem of stars attributable to orbital precession, $W_{G}$ is the gravitational energy of interaction among these stars,

$$
\left|W_{G}\right|=2 \pi^{2} G \int_{0}^{a} \int_{0}^{a} \rho_{l}(r) \rho_{l}\left(r^{\prime}\right) J_{0}\left(r, r^{\prime}\right) d r d r^{\prime},
$$

where we assumed that the disk under consideration consists of "needles" of the same length $2 a$, i.e. all the stars have the same energy $E=E_{0} ; \rho_{l}=r \sigma(r)$ is the quantity proportional to the linear mass density of a needle, while $\sigma(r)$ is the usual surface density; $J_{0}$ is obtained (for $m=0$ ) from a more general expression:

$$
J_{m}(x, y)=\frac{1}{\pi} \int_{0}^{\pi} \frac{\cos (m \alpha) d \alpha}{\sqrt{x^{2}+y^{2}-2 x y \cos (\alpha)}} .
$$

Here we are not interested in a specific value of $\overline{t_{c r}}$.

In the case being considered (large values of $M_{h} / M_{d}$ ) we deal with the version of the radial orbit instability when star orbits as a whole (rather than individual stars) are the elementary objects. In such a case, the exact instability condition can be derived (e.g., Polyachenko 1992). It turns out that this condition is reduced to the intuitional form $\bar{t}<\overline{t_{c r}}$ by replacing $J_{0}\left(r, r^{\prime}\right)$ in the expression (7) for the disk potential energy into $J_{2}\left(r, r^{\prime}\right)$.

\section{References}

Araki, S. 1985, PhD thesis MIT

Combes, F., Debbasch, F., Friedli, D. \& Pfenniger, D. 1990, A\&A, 233, 82

Fridman, A. M. \& Polyachenko, V. L. 1984, Physics of Gravitating Systems, New York: Springer-Verlag

Goldreich, P. \& Lynden-Bell, D. 1965, MNRAS, 130, 125

Julian, W. H. \& Toomre, A. 1966, ApJ, 146, 810

Kulsrud, R., Mark, J. W.-K., \& Caruso, A. 1971, ApJS, 14, 52

Landau, L. D. 1946, Zh. Eksp. Teor. Fiz., 16, 574

Merritt, D. \& Sellwood, J. A. 1994, submitted to ApJ

Ostriker, J. P. \& Peebles, P. J. E. 1973, ApJ, 186, 467

Pasha, I. I. \& Polyachenko, V. L. 1994, MNRAS, 266, 92

Polyachenko, V. L. 1989, Sov. Astron. Lett., 15, 385

Polyachenko, V. L. 1990, in Dynamics of Astrophysical Disks, J. A. Sellwood, Cambridge: Cambridge Univ. Press

Polyachenko, V. L. 1992, Sov. Astron., 69, 10

Polyachenko, V. L. 1994, in Physics of the Gaseous and Stellar Disks of the Galaxy, I. King, San Francisco: ASP, 103 
Polyachenko, V. L. \& Polyachenko, E. V. 1994, Sov. Astr. Lett., 20, 491

Polyachenko, V. L. \& Polyachenko, E. V. 1995, Sov. Astr. Lett., in press

Polyachenko, V. L. \& Polyachenko, E. V. 1995, Sov. Astron., in press

Polyachenko, V. L. \& Shukhman, I. G. 1977, Sov. Astr. Lett., 3, 254

Polyachenko, V. L. \& Shukhman, I. G. 1979, Sov. Astron., 56, 957

Polyachenko, V. L. \& Strelnikov, A. V. 1989, Astron. Tsirk., 5, 3

Polyachenko, V. L. \& Strelnikov, A. V. 1995, Sov. Astron., in press

Raha, N., Sellwood, J. A., James, Q. A., \& Kahn, F. D. 1991, Nature, 325, 411

Sellwood, J. A. \& Wilkinson, A. 1993, Rep. Prog. Phys., 56, 173

Strelnikov, A. V. 1989, diploma work, Moscow State University

Toomre, A. 1966, in Geophys. Fluid Dynamics, 111

Toomre, A. 1981, in Structure and Evolution of Normal Galaxies, S. M. Fall \& D. Lynden-Bell, Cambridge: Cambridge Univ. Press, 111 